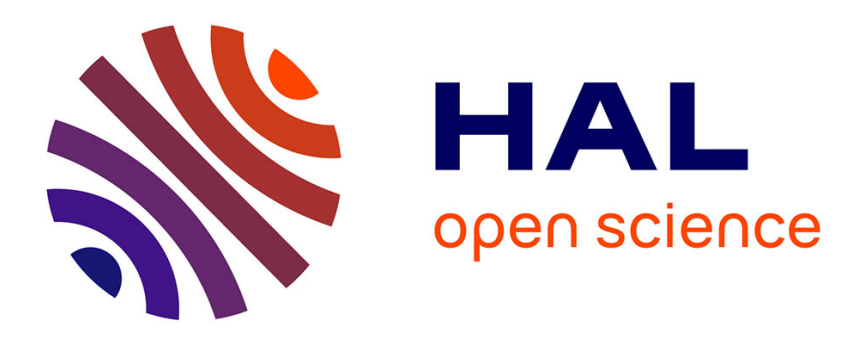

\title{
Influence of Fermi-Dirac Statistics on Collector Current of npn SiGe HBT at Low Temperatures
}

\author{
S. Sokolic, S. Amon
}

\section{To cite this version:}

S. Sokolic, S. Amon. Influence of Fermi-Dirac Statistics on Collector Current of npn SiGe HBT at Low Temperatures. Journal de Physique IV Proceedings, 1996, 06 (C3), pp.C3-131-C3-136. 10.1051/jp4:1996320 . jpa-00254238

\section{HAL Id: jpa-00254238 https://hal.science/jpa-00254238}

Submitted on 1 Jan 1996

HAL is a multi-disciplinary open access archive for the deposit and dissemination of scientific research documents, whether they are published or not. The documents may come from teaching and research institutions in France or abroad, or from public or private research centers.
L'archive ouverte pluridisciplinaire HAL, est destinée au dépôt et à la diffusion de documents scientifiques de niveau recherche, publiés ou non, émanant des établissements d'enseignement et de recherche français ou étrangers, des laboratoires publics ou privés. 


\title{
Influence of Fermi-Dirac Statistics on Collector Current of npn SiGe HBT at Low Temperatures
}

\author{
S. Sokolić and S. Amon \\ Faculty of Electrical Engineering, University of Ljubljana, Tržaška 25, 1000 Ljubljana, Slovenia
}

\begin{abstract}
The doping concentration in the base of npn SiGe HBTs operating at low temperatures should be high to prevent carrier freeze-out and to assure low base resistance. As a consequence, the majority carrier concentration in the base is determined with Fermi-Dirac statistics. The influence of Fermi-Dirac statistics on collector current of $n p n$ SiGe HBTs at low temperatures is analyzed in this work. It is shown that taking Fermi-Dirac statistics into account instead of Boltzmann statistics results in lower collector current. Therefore, Fermi-Dirac statistics should be taken into account for analysis and optimization of these devices. Analytical modelling of effects induced by consideration of Fermi-Dirac statistics is investigated. Based on proposed effective Ge-induced bandgap narrowing with approximately linear dependence on $\mathrm{Ge}$ fraction, the accurate analytical modelling of electron transport in degenerately doped base with trapezoidal Ge profile is presented for the first time.
\end{abstract}

\section{INTRODUCTION}

As a consequence of bandgap narrowing in the base due to Ge and alloying, SiGe HBTs are well suited also for low-temperature operation $[1,2]$. It has been recently demonstrated that SiGe HBTs can achieve better performances at LNT $(77 K)$ than at RT (300K) [3].

The importance of Fermi-Dirac (FD) statistics has been traditionally related to the operation of highly doped emitters of bipolar transistors. Since the doping concentration in the base of SiGe HBTs operating at low temperatures should be high enough to prevent carrier freeze-out and to assure low base resistance, FD statistics instead of Boltzmann (B) statistics should be taken into consideration for analysis and optimization of low temperature base transport properties of SiGe HBTs. Existing analytical expressions for the collector current $\left(I_{C, S_{i G e}}\right)$ and related properties of SiGe HBTs are generally based on $B$ statistics $[1,2,4]$. For this reason such expressions seem not to be appropriate for low-temperature analyses of SiGe HBTs [1,2]. In this work we analyze the influence of FD statistics in the base of SiGe HBT, and propose a new approach to the analytical modelling of the collector current in SiGe HBT, taking FD statistics critically into account.

\section{PHYSICS OF DEGENERATELY DOPED SiGe BASE}

\subsection{Minority carrier concentration}

The main effect of FD statistics in p-type SiGe base is an increase of hole Fermi level compared to the hole Fermi level determined with B statistics. As a consequence, the electron concentration is decreased 
in comparison with electron concentration determined with B statistics. An effective bandgap narrowing $\left(\Delta E_{\text {deg }}\right)$ can be defined [5], taking this effect into account:

$$
\Delta E_{\text {deg }}=k T \ln \left(\frac{N_{A}^{-}}{N_{V, S i G e}(F D)}\right)-k T \mathrm{G}_{1 / 2}\left(\frac{N_{A}^{-}}{N_{V, S i G e}(F D)}\right),
$$

where $\mathrm{G}_{1 / 2}$ is inverse function of Fermi-Dirac integral of order $1 / 2 . N_{V, S i G e}(F D)$ is the effective density of states in the valence band taking FD statistics into account, where it is assumed that the consideration of FD statistics introduces explicit doping dependence of effective density of states (via doping dependence of hole effective mass). Since appropriate analytical approximations for $G_{1 / 2}$ exist, (1) represents explicit analytical expression for $\Delta E_{\text {deg. }}$.

In addition to $\Delta E_{d e g}$, an effective bandgap narrowing due to lower effective density of states in SiGe compared to the density of states in silicon $\left(\Delta E_{D o s}\right)$ is also influenced by FD statistics, which is a consequence of doping dependence of effective density of states in the valence band. The difference between $\Delta E_{D O S}$ considering FD statistics and $\Delta E_{D O S}$ considering B statistics $\left(\Delta E_{D O S, F D}=\Delta E_{D O S}-\Delta E_{D O S, B}\right)$ can be accordingly defined:

$$
\Delta E_{D O S, F D}=k T \ln \left(\frac{N_{C, S i G e} N_{V, S i G e}(F D)}{N_{C, S i}^{0} N_{V, S i}^{0}}\right)-k T \ln \left(\frac{N_{C, S i G e} N_{V, S i G e}(B)}{N_{C, S i}^{0} N_{V, S i}^{0}}\right)=k T \ln \left(\frac{N_{V, S i G e}(F D)}{N_{V, S i G e}(B)}\right) .
$$

where $N_{V, S i G e}(B)$ represent doping independent effective density of states, which can be obtained as a lowdoping limit value of $N_{V, S i G e}(F D)$. Both effects $\left(\Delta E_{d e g}\right.$ and $\left.\Delta E_{D O S, F D}\right)$ depend significantly on the effective density of states in the valence band $\left(N_{V, S i G e}\right)$, which is determined with hole effective mass $\left(m_{p}{ }^{*}\right)$. As $m_{p}{ }^{*}$ is a function of temperature $(T)$, doping concentration $\left(N_{A}^{-}\right)$and $\mathrm{Ge}$ fraction $\left(x_{G e}\right)[6,7]$, the magnitudes of $\Delta E_{\text {deg }}$ and $\triangle E_{D O S, F D}$ depend on $T, N_{A}^{-}$and $x_{G e}$.

$\Delta E_{\text {deg }}, \Delta E_{D O S . F D}$ and $\Delta E_{\text {deg }}+\Delta E_{D O S, F D}$ are presented on Fig.1. The calculations are based on the model for $m_{p}{ }^{*}$ from [7]. As can be seen, $\Delta E_{d e g}+\Delta E_{D O S, F D}$ is negative and its absolute magnitude increases rapidly with increasing doping concentration, increasing $\mathrm{Ge}$ fraction and decreasing temperature.

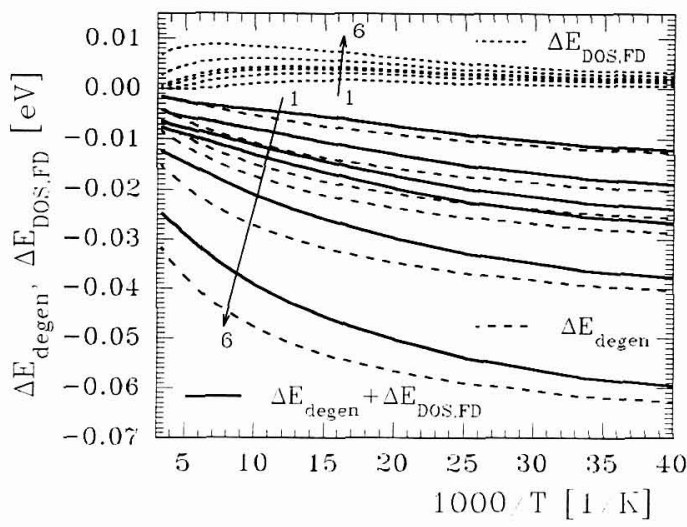

(a)

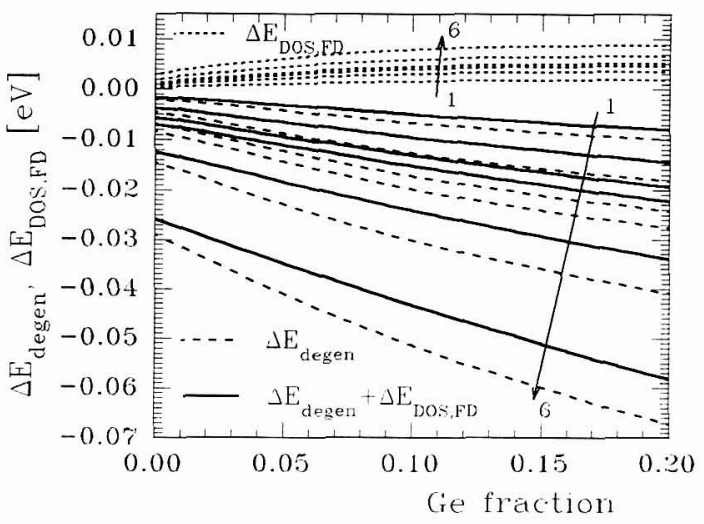

(b)

Figure 1: $\Delta E_{\text {deg }}, \Delta E_{D O S, F D}$ and $\Delta E_{\text {deg }}+\Delta E_{D O S, F D}$ calculated from (1) and (2): (a) as a function of reciprocal temperature $\left(x_{G e}=0.1\right)$; (b) as a function of $\mathrm{Ge}$ fraction $(T=77 \mathrm{~K})$. Curves 1-6 correspond to different doping concentrations: $2 \cdot 10^{18} \mathrm{~cm}^{-3}$, $5 \cdot 10^{18} \mathrm{~cm}^{-3}, 8 \cdot 10^{18} \mathrm{~cm}^{-3}, 10^{19} \mathrm{~cm}^{-3}, 2 \cdot 10^{19} \mathrm{~cm}^{-3}$ and $5 \cdot 10^{19} \mathrm{~cm}^{-3}$. 


\subsection{Collector current}

It can be shown that Kroemer's relation for minority carrier current [8] is valid also for degenerate conditions in the base. If it is assumed that the collector current can be approximated with the electron current in the base and that recombinations in the base can be neglected, the collector current in SiGe HBT with degenerately doped base is determined with (3):

$$
I_{C, S i G e}=A q n_{i, S i}^{02} D_{n, S i} \exp \left(\frac{q V_{B E}}{k T}\right)\left(\int_{0}^{W_{B}} \exp \left(-\frac{\Delta E_{g, G e}+\Delta E_{g, h d}+\Delta E_{D O S, B}+\Delta E_{D O S, F D}+\Delta E_{d e g}}{k T}\right) \frac{N_{A}^{-} d x}{\eta}\right)^{-1}
$$

where $n_{i, S i}^{0}$ is intrinsic concentration in silicon, $\eta$ is $D_{n, S i G e} / D_{n, S i}[9], \Delta E_{g, G e}$ is bandgap narrowing caused by $\mathrm{Ge}$ and alloying, $\Delta E_{g, h d}$ is doping induced bandgap narrowing and other symbols have their usual meaning. Equation (3) has the same form as the corresponding expression for the case of B statistics only exponential term under integral is modified by adding $\Delta E_{\text {deg }}$ and $\Delta E_{D O S, F D}$.

The ratio of collector current calculated by means of FD and $B$ statistics is presented on Fig. 2 . As can be seen, the use of B statistics instead of FD statistics results in significant overestimation of collector current at low temperatures, which increases rapidly with increasing doping concentration. As a consequence, the use of Boltzmann statistics appears to be inappropriate for SiGe HBT modelling at $77 K$. Box Ge profile is more sensitive to the influence of FD statistics than trapezoidal Ge profile for the same $\mathrm{Ge}$ dose. This is an expected result, because the collector current is influenced mainly by the bandgap narrowing at the emitter side of the base and the consideration of FD statistics is more influential at higher Ge fractions.

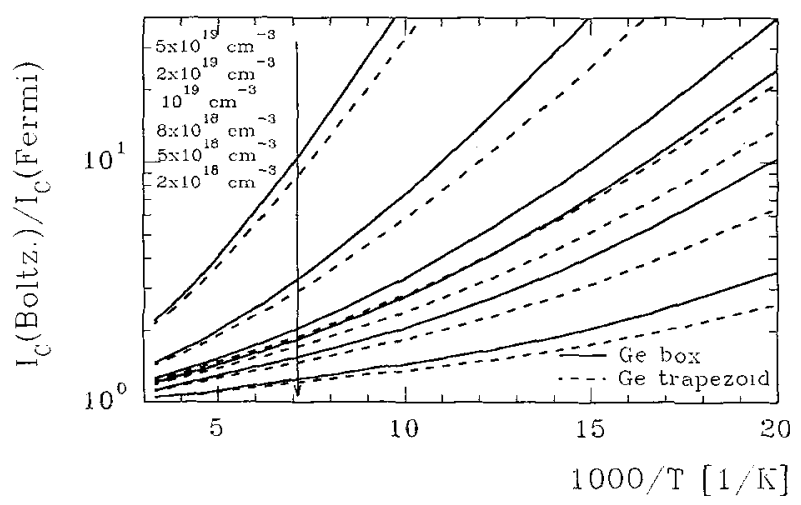

Figure 2: Ratio of $I_{C, S i G e}$ calculated with B statistics and FD statistics as a function of reciprocal temperature. Solid curves correspond to box Ge profile $\left(x_{G e}=0.06\right)$ and dashed curves correspond to trapezoidal Ge profile $\left(0.03<x_{G e}<0.09\right)$.

\subsection{Current gain ratio}

As can be seen from Fig.1, the consideration of FD statistics is more influential in SiGe than in silicon. Therefore, the enhancement of the current gain in SiGe HBT with respect to the current gain in Si BJT with the same doping profile and the same geometry $\left(\beta_{S i G e} / \beta_{S i}\right)$ is also influenced by consideration of FD statistics. The results presented on Fig. 3 demonstrate that $\beta_{S i G e} / \beta_{S i}$ at low temperatures determined with FD statistics is not as high as $\beta_{S i G e} / \beta_{S i}$ predicted by B statistics. The difference is especially high in the case of box Ge profile and high doping concentration in the base. 


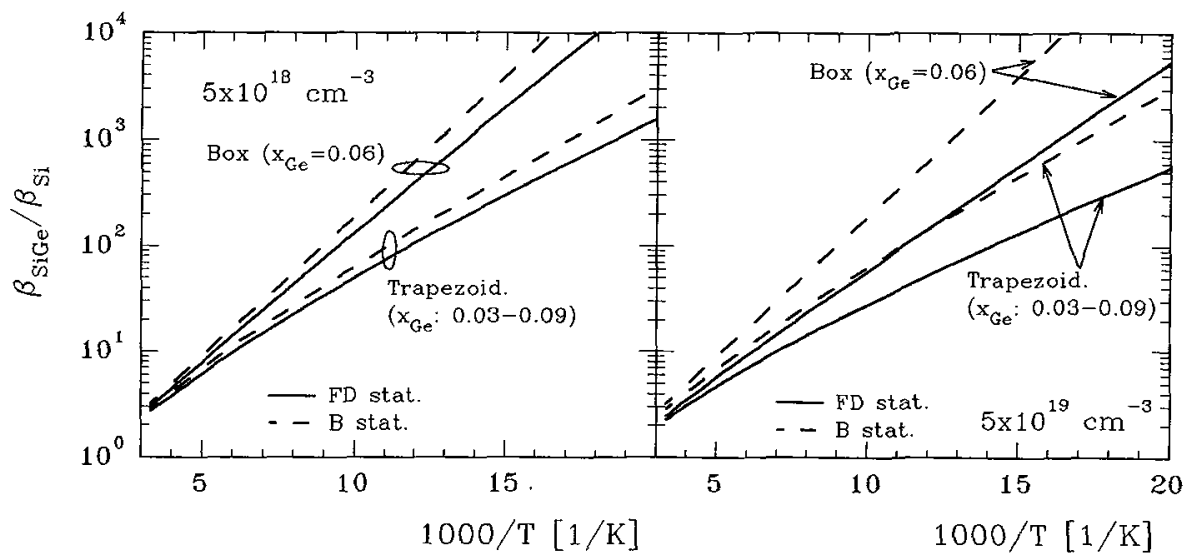

(a)

(b)

Figure 3: The ratio of current gain in SiGe HBT and Si BJT with the same doping profile and the same geometry as a function of reciprocal temperature. (a) $N_{A}=5 \cdot 10^{18} \mathrm{~cm}^{-3}$. (b) $N_{A}^{-}=5 \cdot 10^{19} \mathrm{~cm}^{-3}$.

\section{EFFECTIVE Ge-INDUCED BANDGAP NARROWING}

In the case of uniform doping and box Ge profile in the base, eq. (3) can be solved analytically. By means of analytical models for $\Delta E_{d e g}$ and $\Delta E_{D O S, F D}(1,2)$, the derivation of analytical expression for the electron current in the degenerately doped base is straightforward. In the case of trapezoidal Ge profile, position dependence of Ge-induced effects $\left(\Delta E_{g, G e}, \Delta E_{D O S, B}, \Delta E_{D O S, F D}, \Delta E_{D O S, B}, D_{n, S i G e}\right)$ should be taken into account and eq. (3) cannot be solved analytically without simplifications (integral of $\exp \left(\mathrm{G}_{1 / 2}\right)$ in (3) cannot be solved analytically). An analytical expression for $I_{C}$ in the case of B statistics $\left(\triangle E_{D O S, F D}\right.$ and $\Delta E_{\text {deg }}$ are neglected) was presented in [4], where position averaged quantities of $\Delta E_{D O S, B}$ and $D_{n, S i G e}$ were introduced and $\exp \left(-\Delta E_{g, G e} / k T\right)$ was integrated.

To account for the effect of FD statistics, we introduce the effective Ge-induced bandgap narrowing $\left(\Delta E_{g, G e, e f f}\right)$ :

$$
\Delta E_{g, G e, e f f}=\Delta E_{g, G e}+\Delta E_{D O S, B}+\Delta E_{D O S, F D}+\Delta E_{d e g}+k T \ln (\eta)
$$

Beside $\Delta E_{g, G e}$ and the effects induced by FD statistics, $\Delta E_{D O S, B}$ and $\eta$ are included in $\Delta E_{g, G e, e f f}$ as well. As can be seen from Fig. $4 \mathrm{a}, \Delta E_{g, G e, \text { eff }}$ is lower than $\Delta E_{g, G e}$ due to FD statistics and decreased effective density of states. FD statistics therefore lower the beneficial Ge-induced bandgap narrowing in the base. Since $\Delta E_{g, G e, e f}$ is approximately linear function of Ge fraction, an effective trapezoidal Ge profile can be found for every grown trapezoidal Ge profile, doping concentration in the base and temperature (Fig. $4 \mathrm{~b}$ ). Since the trapezoidal Ge profile can be treated analytically $[2,4]$, the collector current in SiGe HBT with degenerately doped base and trapezoidal Ge profile can be modelled analytically by means of $\Delta E_{g, G e, e f f}$. In this approach it is necessary to evaluate $\Delta E_{g, G e, \text { eff }}$ only at both edges of the base. The collector current then reads:

$$
I_{C, S i G e}=\frac{A q n_{i, S i}^{02} D_{n, S i}}{W_{B} N_{A}^{-}} \exp \left(\frac{q V_{B E}}{k T}\right) \frac{\left(\Delta E_{g, G e, e f f}\left(W_{B}\right)-\Delta E_{g, G e, e f f}(0)\right) \exp \left(\frac{\Delta E_{g, G e, e f f}+\Delta E_{g, h d}}{k T}\right)}{k T\left(1-\exp \left(-\frac{\Delta E_{g, G e, e f f}\left(W_{B}\right)-\Delta E_{g, G e, e f f}(0)}{k T}\right)\right)} .
$$




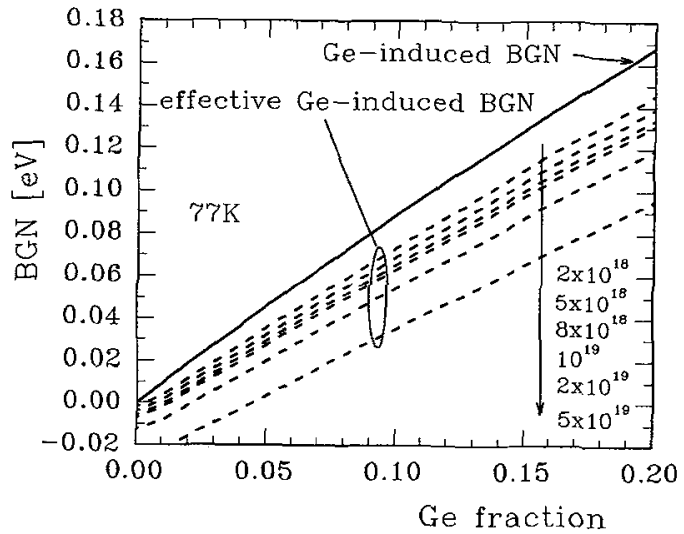

(a)

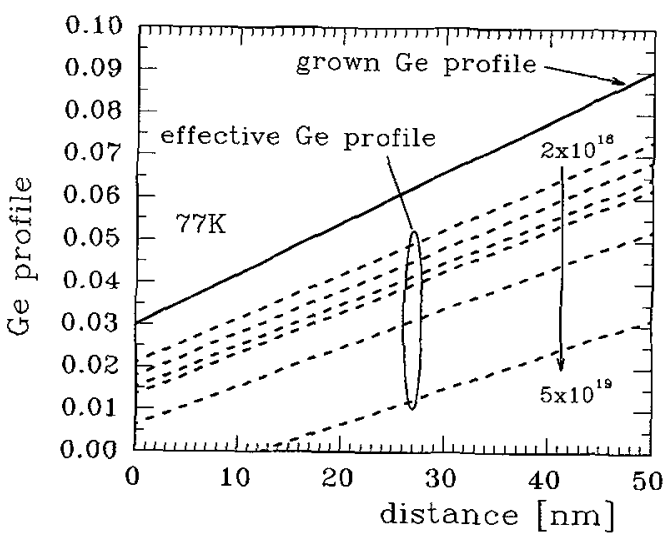

(b)

Figure 4: (a) Ge-induced bandgap narrowing and the effective Ge-induced bandgap narrowing as a function of Ge fraction calculated for different doping concentrations $(T=77 K)$. (b) Grown trapezoidal Ge profile $\left(0.03 \leq x_{G e} \leq 0.09\right)$ and effective Ge profiles in the base of SiGe HBT obtained for different doping concentrations at $T=77 \mathrm{~K}$.

The ratio of approximated collector current (eq. (5)) and the exact solution for collector current (exact integration of (3)) is presented on Fig.5. As can be seen, $\Delta E_{g, G e, e f f}$ approach is in excellent agreement with exact numerical integration of (3) over the entire range of temperatures. As a consequence, introduction of $\Delta E_{g, G e, \text { eff }}$ represents an elegant approach to the accurate analytical modelling of carrier transport in degenerately doped base with trapezoidal Ge profiles. For a comparison, the collector current was calculated also by means of position averaged $\Delta E_{D O S, B}$ and $\eta$, while other position dependent terms in (3) were integrated numerically. As can be seen from Fig.5, this approach is inferior to $\Delta E_{g, G e, e f f}$ approach in the entire temperature range and its accuracy decreases with decreasing temperature.

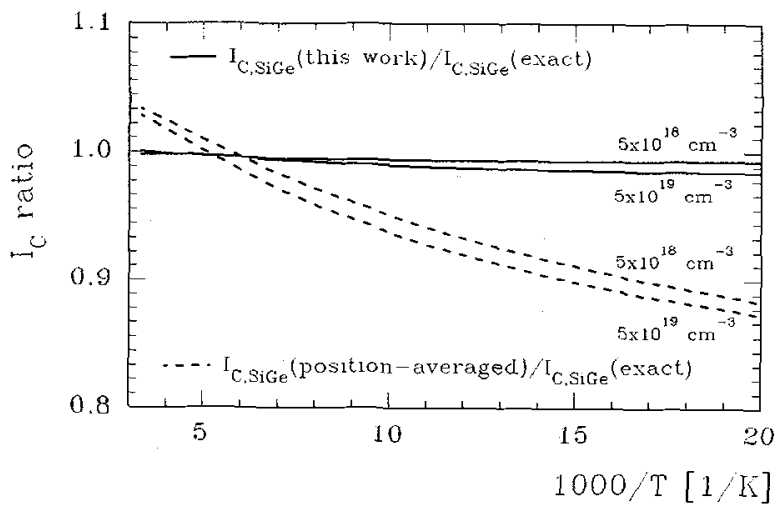

Figure 5: The ratio of collector current (trapezoidal Ge profile $\left(0.03 \leq x_{G e} \leq 0.09\right)$ ) calculated with approximate and exact solution of (3). Solid curves represent the approximation proposed in this work (eq. (5)). Dashed curves represent the approximation where $\Delta E_{D O S, B}$ and $\eta$ are position averaged and other position dependent terms in (3) (exp (- $\Delta E_{g, G e^{-}-\Delta E_{d e g}-}$ $\left.\Delta E_{D O S, F D}(k T)\right)$ are integrated numerically. 


\section{CONCLUSIONS}

The influence of Fermi-Dirac statistics on the collector current of npn SiGe HBT at low temperatures, together with the possibilities for analytical modelling of this effect are studied in details.

It is demonstrated that the consideration of Boltzmann statistics overestimates the collector current of $n p n$ SiGe HBT at low temperatures. Consequently, FD statistics should be taken into consideration for accurate modelling of base transport properties of SiGe HBTs operating at 77K for all base doping concentrations and $\mathrm{Ge}$ fractions of interest. As a consequence of its dependence on Ge fraction, the consideration of FD statistics affects also the relative improvement of SiGe HBTs with respect to the $\mathrm{Si}$ BITs. It is shown that FD statistics cause lower enhancement of current gain in SiGe HBTs compared to the current gain in Si BJTs $\left(\beta_{S i G e} / \beta_{S i}\right)$, than the enhancement predicted by B statistics.

The effective Ge-induced bandgap narrowing was introduced, consisting of bandgap narrowing due to $\mathrm{Ge}$ and alloying, effect of decreased density of states in SiGe, Ge dependence of minority carrier diffusion constant, and effects induced explicitly by FD statistics. By means of the effective Ge-induced bandgap narrowing, accurate analytical modelling of collector current of SiGe HBT with degenerately doped base and trapezoidal Ge profile is presented for the first time. According to the importance of FD statistics for low temperature performances of SiGe HBTs, the results of this work could be important for analysis and optimization of low-temperature properties of SiGe HBTs.

\section{Acknowledgement}

This work was partially sponsored by the Ministry of Science and Technology of the Republic of Slovenia.

\section{References}

[1] Cressler J. D., "Status and trends in the cryogenic operation of SiGe bipolar technology", Symp. on Low Temp. Electron. and High Temp. Supercond., Reno, Nevada 21-26 May 1995, C. L. Claeys, S. I. Raider, R. K. Kirschman and W. D. Brown Eds. (ECS PV 95-9, The Electrochemical Society, Inc., Pennington) pp. 159-177.

[2] Cressler J. D., Comfort J. H., Crabbé E. F., Patton G. L., Stork J. M. C., Sun J. Y.-C., Meyerson B. S., IEEE Trans. Electron Dev. 40 (1993) 525-541.

[3] Cressler J. D., Crabbé E. F., Comfort J. H., Sun J. Y.-C., Stork J. M. C., IEEE Electron Dev. Lett. 15 (1994) 472-474.

[4] Harame D. L., Comfort J. H., Cressler J. D., Crabbé E. F., Sun J. Y.-C., Meyerson B. S., Tice T., IEEE Trans. Electron Dev. 42 (1995) 455-468.

[5] Sokolić S., Amon S., "Modelling Heavy Doping Effects for Low Temperature Device Simulations", First European Workshop on Low Temperature Electronics, Grenoble 19 June - 1 July 1994, G. Ghibaudo and F. Balestra Eds. (J. Phys. IV, Les Editions de Physique, Les Ulis, 1994), pp. C6-133 - C6-138.

[6] Manku T., Nathan A., J. Appl. Phys. 69 (1991) 8414-8416.

[7] Sokolić S., Amon S., accepted for presentation at WOLTE 2.

[8] Kroemer H., Solid-St. Electron. 28 (1985) 1101-1103.

[9] Decoutere S., Poortmans J., Deferm L., Nijs J., Solid-St. Electron. 38 (1995) 157-162. 